\title{
Fabrication and characterization of a NIR-FIR dichroic for the infrared interferometer BETTII
}

\section{Arnab Dhabal, Stephen A. Rinehart, Robert F. Silverberg, Dale Fixsen, John E. Mentzell, et al.}

Arnab Dhabal, Stephen A. Rinehart, Robert F. Silverberg, Dale Fixsen, John E. Mentzell, Todd Veach, Richard Kasica, Jessie Zhang, Carole Tucker, Peter Ade, "Fabrication and characterization of a NIR-FIR dichroic for the infrared interferometer BETTII," Proc. SPIE 10708, Millimeter, Submillimeter, and Far-Infrared Detectors and Instrumentation for Astronomy IX, 107083I (9 July 2018); doi: 10.1117/12.2312146

Event: SPIE Astronomical Telescopes + Instrumentation, 2018, Austin, Texas, United States 


\title{
Fabrication and characterization of a NIR-FIR dichroic for the infrared interferometer BETTII
}

\author{
Arnab Dhabal ${ }^{\mathrm{a}, \mathrm{b}}$, Stephen A. Rinehart ${ }^{\mathrm{a}}$, Robert F. Silverberg ${ }^{\mathrm{a}}$, Dale Fixsen ${ }^{\mathrm{a}}$, John E. \\ Mentzell $^{\mathrm{a}}$, Todd Veach ${ }^{\mathrm{a}}$, Richard Kasica ${ }^{\mathrm{c}}$, Jessie Zhang ${ }^{\mathrm{c}}$, Carole Tucker ${ }^{\mathrm{d}}$, and Peter Ade ${ }^{\mathrm{d}}$ \\ anASA Goddard Space Flight Center, Greenbelt, MD 20770, USA \\ ${ }^{\mathrm{b}}$ Department of Astronomy, University of Maryland, College Park, MD 20741, USA \\ cNational Institute of Standards and Technology, Gaithersburg, MD 20899, USA \\ ${ }^{\mathrm{d} S c h o o l}$ of Physics and Astronomy, Cardiff University, Cardiff, CF24 3AA, UK
}

\begin{abstract}
BETTII is a balloon-borne far infra-red (FIR: 30-100 $\mu \mathrm{m}$ ) interferometer that also uses a near-infrared (NIR: 1-2.5 $\mu \mathrm{m}$ ) channel for fine pointing sensing using stars. We have developed an inductive grid dichroic to divide the incoming beam into two components, by reflecting FIR light and transmitting NIR light. The dichroic is fabricated using focused electron beam technology to produce a $1 \mu \mathrm{m}$ period, $100 \mathrm{~nm}$ width metal grid on a sapphire substrate in order to have high reflectance for FIR wavelengths. Here we discuss the design and the detailed manufacturing process for such a dichroic. The transmission and reflectance characteristics are also presented. We discuss them in context of the BETTII requirements.
\end{abstract}

Keywords: spatio-spectral interferometry, far infra-red, delay line, capacitive sensor, interferometric simulation, metrology, alignment

\section{INTRODUCTION}

Star formation occurs in dust obscured regions. The dust typically has temperatures in the range 25-300 K, which are best observed in the mid to far-infrared wavelengths. The earth's atmosphere is almost opaque at these wavelengths; so their study requires observatories at a high altitude from the earth's surface or in space. In the last few decades, the development of infrared instruments for space-based and balloon-borne astronomical observatories has been possible due to advances in cryogenic performance ${ }^{1}$ and detector technologies for infrared wavelengths. ${ }^{2}$ These advancements have been accompanied by a requirement for filters for these wavelengths.

Different infrared astronomical instruments have different filter requirements, which are met by a variety of filter designs and technologies. Metal mesh filters have been used to reduce the radiative thermal loading on cryostats and detectors, and to reduce the out-of-band high frequency spectral leakage. ${ }^{3,4}$ They have been used as intensity beamsplitters on the SPIRE instrument of the Herschel observatory. ${ }^{5}$ Frequency selective surfaces (FSS) such as slots in metal sheets have been used to get transmission between 20-60 $\mu \mathrm{m}$ for planetary spectroscopy. ${ }^{6}$ Restrahlen crystal filters were used as a dichroic beam-splitters for the short wavelength spectrometer (SWS) on Infrared Space Observatory (ISO). ${ }^{7}$ Multilayer interference filters have also been developed, for example the CdTe Q-band filter to be used as a wide bandpass filter on the Mid-infrared instrument (MIRI) of the James Webb Space Telescope (JWST). ${ }^{8}$

BETTII (Balloon Experimental Twin Telescope for Infrared Interferometry) is a balloon-borne double-Fourier interferometer capable of Michelson interferometry and simultaneous Fourier Transform Spectroscopy in the FIR wavelengths of 30-100 $\mu \mathrm{m}$ (Figure 1). The optics of BETTII collects light from two apertures 8 meters apart and combines them, while modulating the optical path lengths to produce interferometric fringes at the FIR detectors. The FIR optics train requires multiple filters: thermal blockers to reduce thermal loading, edge pass filters to reduce out-of-band radiation, dichroics to separate the FIR light into multiple observing bands, and a beam-splitter to combine radiation from the two arms (transmitted light from one arm, with reflected light

Author contact: Arnab Dhabal, arnab.dhabal@nasa.gov

Millimeter, Submillimeter, and Far-Infrared Detectors and Instrumentation for Astronomy IX, edited by Jonas Zmuidzinas, Jian-Rong Gao, Proc. of SPIE Vol. 10708, 107083I (C) 2018 SPIE · CCC code: 0277-786X/18/\$18 · doi: 10.1117/12.2312146 


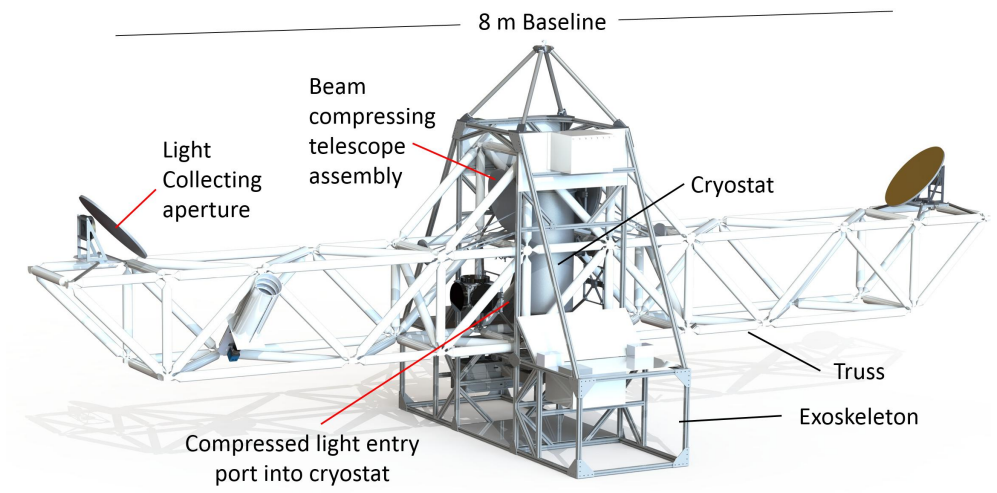

Figure 1. BETTII model showing the truss supporting the external optics and the exoskeleton supporting the electronics. The external optics comprise the light collecting apertures and beam compressing telescope assemblies on both arms of the interferometer. The compressed light enters the cryostat at the center from the two arms. The cryostat houses the NIR optics and the FIR optics bench (shown in Figure 2).

from the other). All these filters for FIR wavelengths are based on Ade et al. (2006), ${ }^{9}$ and their performance is discussed in Dhabal et al. (2016). ${ }^{10}$

In addition to these filters, the BETTII design requires another dichroic for each arm to separate the FIR science band $(30-100 \mu \mathrm{m})$ from the NIR band $(1-2.5 \mu \mathrm{m})$, which is used for fine pointing control. The NIR light, focused on a Teledyne H4RG detector ${ }^{11}$ is used to locate sources in the FOV, and drive a tip-tilt mirror in a closed control loop to center the observing target on both the NIR and FIR detectors. ${ }^{12,13}$ Inside the BETTII cryostat, this dichroic is the first optic, which divides the $25 \mathrm{~mm}$ diameter beam into its FIR and NIR components (Figure 2). In the following sections, we motivate the inductive grid design of this dichroic and discuss its fabrication, which involves the e-beam lithography technique to attain the smaller length scales involved in comparison to the filters in the FIR optics train. We present the microscope inspection results, and the transmission and reflectance properties of the fabricated dichroic in the final section.

\section{DICHROIC DESIGN}

We considered a wide range of possible filters that have been used in NIR and FIR wavelengths such as reststrahlen filters, powder filters, interference thin-film filters and metal mesh filters. Interference filters involving the use of multi-layer dielectric coatings to create the dichroic response is problematic. This is because the coating layers are dielectrics which have phonon absorptions in the band that we are considering. Additionally, the layers would need to be thick and hence suffer from differential thermal contraction and fracture as they are cooled. Crystalline materials were found to be too restrictive in their reststrahlen band reflectance to be used in this application.

Since the FIR science bands are well separated from the NIR control band, we can take advantage of the relatively slow switch criteria. Metal grids are ideal for this application because even a single mesh has good reflectance when the mesh period is much smaller than the shortest wavelength we need to reflect; so a $1 \mu \mathrm{m}$ period mesh would be an excellent reflector at $30 \mu \mathrm{m}$. The grids operate according to the same principle as a large radio telescope constructed using a crude wire mesh that lightens the structure without loss of reflectance for the very long wavelengths. Such a mesh would also display maximum transmission when the wavelength is comparable to or less than the mesh period and it would not be in the diffraction regime.

Two-dimensional metal meshes and slots have been studied since the mid-20th century as filters for sub-mm and FIR wavelengths. Bell $(1966)^{14}$ used the inductive grid (orthogonal pattern of narrow wires) as a beamsplitter in a Michelson type Fourier transform spectrometer (FTS). Ulrich $(1967)^{15}$ introduced the equivalent circuit model to obtain the optical transmission properties of a metal mesh and extended the concept to capacitive 


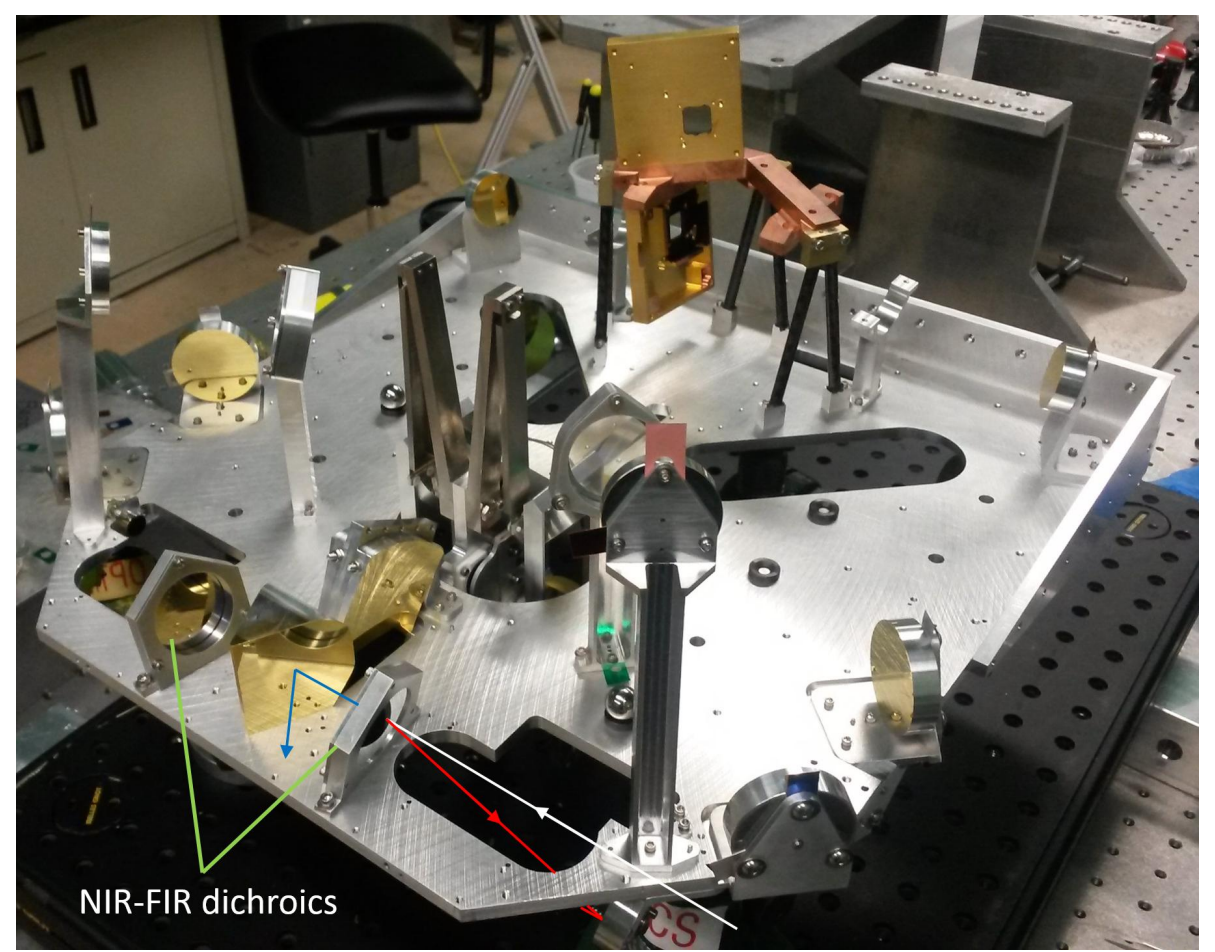

Figure 2. The FIR optics bench showing the location of the NIR/FIR dichroics. The dichroics divide the incoming beam (white) into the NIR band that is transmitted (blue), and the FIR band that is reflected (red). The rest of the optics shown here are part of the FIR optics train. The NIR optics focuses the transmitted beam onto a H4RG array (not shown here).

grids (geometric inverse of inductive grids). The optical properties of the grids depend on the pitch, linewidth and thickness, as has been theoretically determined by the transmission line theory ${ }^{16,17}$ and experimentally verified $^{18,19}$ by multiple researchers. Since the development of the High Frequency Structure Simulator (HFSS) in the late 1980s, it has been extensively used to analyze the electromagnetic properties of such metal mesh structures. $^{9}$

Following modeling work using HFSS which consolidated the concept, we decided to manufacture a dichroic using a single inductive metal mesh with a period $\mathrm{g}=1 \mu \mathrm{m}$. The optimum value of linewidth ' $2 \mathrm{a}$ ' and layer thickness 'd' were found to be $100 \mathrm{~nm}$ and $45 \mathrm{~nm}$ respectively. This in the low a/g and t/g regime $(\sim 0.05)$, which determines the optical properties of the grid. A low value of a/g results in a slow switch from the transmission band to the reflection band. ${ }^{20}$ The bandwidth of the transmission maximum increases with decreasing a/g and $\mathrm{t} / \mathrm{g}$. Effects of Wood anomalies are also lower for low $\mathrm{t} / \mathrm{g}$ values. ${ }^{21}$ The model is however sensitive to incident angles, which shifts the peak transmission wavelength, and changes the depth of a sharp drop in the transmission profile called the 'thickness dip'. ${ }^{19}$

We decided to use e-beam lithography to produce inductive grids of the required quality at the scale of these dimensions. Comparable techniques have been used in the recent past, but for metal slot based FSS fabrication. ${ }^{22,23}$ Other techniques of grid fabrication involve photolithography ${ }^{24}$ and laser ablation, ${ }^{25}$ but neither are suitable for our linewidth and resolution requirements. The inductive grid is made of $\mathrm{Au}$ for high conductivity. Since we are reflecting the FIR, the substrate on which the grid is fabricated must be flat to better than $\lambda / 20$ of $30 \mu \mathrm{m}$-the shortest wavelength reflected. Materials which transmit the optical/NIR light are plentiful but given this flatness requirement we chose to use a crystalline material: C-cut sapphire. This material has good optical properties, can be anti-reflection (AR) coated and processing using Au deposition with electron beam lithography is not an issue. 


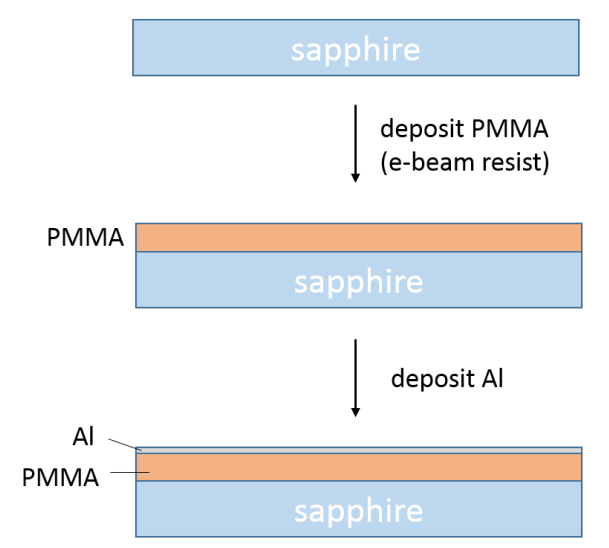

1

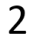

3
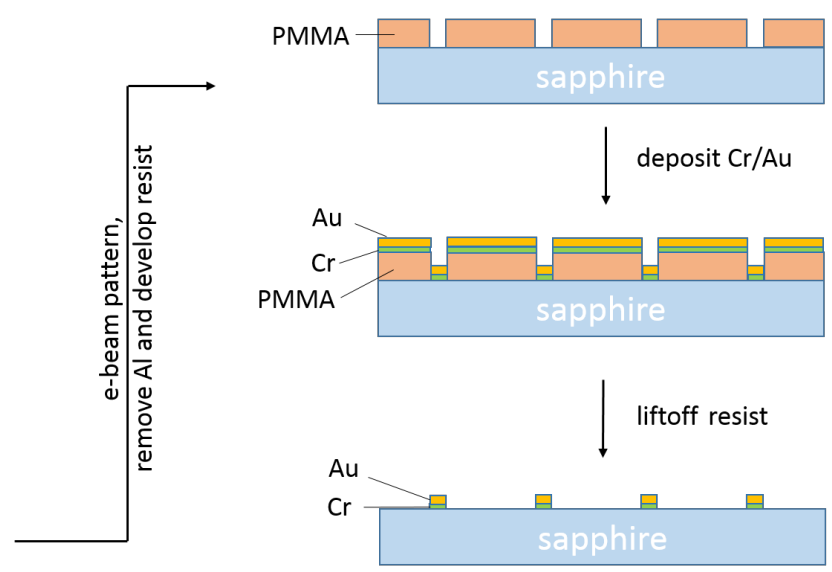

Figure 3. The inductive grid fabrication steps starting from a sapphire substrate. (Layer thicknesses not to scale)

\section{FABRICATION}

We acquired $1 \mathrm{~mm}$ thick $38.1 \mathrm{~mm}$ diameter C-cut sapphire substrates for our application. On one surface, the substrates were $\mathrm{AR}$ coated with multiple layers of $\mathrm{Ta}_{2} \mathrm{O}_{5}+\mathrm{SiO}_{2}$ with $\mathrm{SiO}_{2}$ being the outermost layer to be scratch resistant on one face. This material has $>98 \%$ transmission in our NIR band of 1-2.5 $\mu \mathrm{m}$. We used the opposite surface for fabricating the inductive Au grid.

The grid fabrication process is illustrated in Figure 3 with the steps indicated in the following lines. The sapphire substrates were first cleaned using acetone, methanol, and iso-propyl alcohol (IPA) and allowed to dry at $180^{\circ} \mathrm{C}(1)$. The samples were then coated with poly (methyl methacrylate) or PMMA resist and baked at $140^{\circ} \mathrm{C}$ with a target thickness of $200 \mathrm{~nm}(2)$. This was followed by a thin $5 \mathrm{~nm}$ film of aluminum deposited on top of the resist to be the charge dissipating layer during the writing (3). Samples were exposed using a JEOL JBX-6300 e-beam system operating at $100 \mathrm{kV}$ at a dose of $925 \mu \mathrm{C} / \mathrm{cm}^{2}$. Due to the large area, it took about 30 hours of writing time. After exposure, the aluminum was removed by a 30 second soak in a tetramethylammonium hydroxide (TMAH) based resist developer. After rinsing with water and drying, the PMMA was developed in a solution of methyl isobutyl ketone solvent (MIBK) and IPA in the ratio 1:3 for 60 sec at room temperature (4). This was followed by a blanket deposition of $5 \mathrm{~nm} \mathrm{Cr} / 45 \mathrm{~nm}$ Au using an e-beam evaporator (5). The final step was the removal of resist and lifting off of the metal stack in the non-patterned areas (6). This was performed in n-methyl pyrrolidone at a temperature of $80^{\circ} \mathrm{C}$. Ultrasonic agitation was used at the beginning of the lift-off process and the patterned sapphire substrate was allowed to soak for at least 12 hours.

We first developed the grid on a small region of area $10 \mathrm{~mm} \times 10 \mathrm{~mm}$ using the e-beam lithographic process, and tested the reflectance and transmittance of the samples. The e-beam writing is carried out over unit areas of $1 \mathrm{~mm} \times 1 \mathrm{~mm}$ at a time, and the instrument needs to be calibrated to prevent stitching errors (offset, discontinuity and overlap). After establishing the procedure, we scaled up to develop the grid over a circular area of diameter $30 \mathrm{~mm}$ for the flight filters.

\section{PERFORMANCE}

Preliminary inspection of the dichroic (Figure 4) showed some damage or improper production at the periphery especially at the edges of the $1 \mathrm{~mm}$ pattern squares. Under a microscope, we did not find any stitching defects between the tiles. However, some lift-off problems were identified resulting in metal remaining within some of the grids (see Figure 5). It could be partially improved with additional ultrasonics throughout the liftoff cycle. It could come from non-uniformities in the resist coating which could make liftoff more difficult in those areas.

We inspected the inductive grid using Scanning Electron Microscopy (SEM). As shown in Figure 6, the linewidth is uniform, with variations of $\sim 5 \mathrm{~nm}$ over most of the sample. Only some regions were identified having larger deviations from the target linewidth and pattern (Figure 7). This is caused due to poor adhesion 


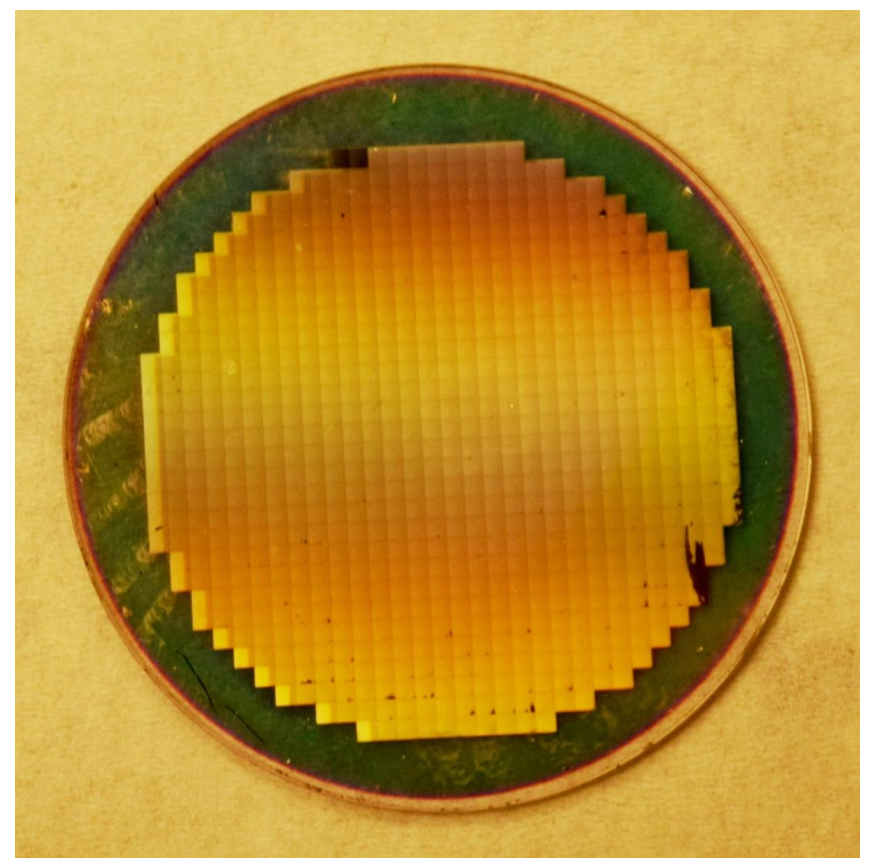

Figure 4. The fabricated dichroic developed on a sapphire substrate of diameter $38.1 \mathrm{~mm}$. The image shows the tiling due to the unit pattern covering a circle of diameter $30 \mathrm{~mm}$. Some damages are evident near the periphery, but most of them are outside the beam footprint of diameter $25 \mathrm{~mm}$.

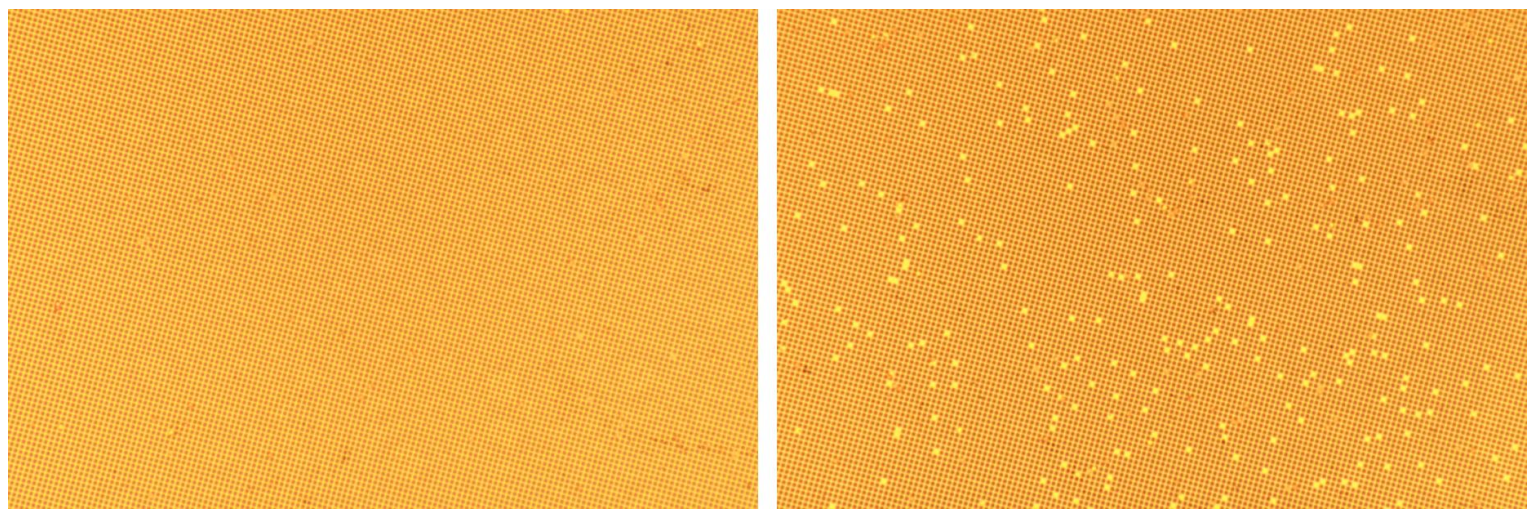

Figure 5. Zoomed in images of the dichroic: (left) region with very few lift-off problems and (right) area showing a higher concentration of lift-off errors. Images taken using Nikon optical microscope at 100X zoom.

of the resist to the substrate in these regions. The defective squares lifted during development (between steps (3) and (4) in Figure 3) and then dropped back into place after drying with a slight rotation. So on Au deposition, it results in variable linewidths locally. Overall, this affects less than $1 \%$ of the squares. Using Atomic Force Microscopy (AFM), we determined that the thickness of the Au grid is also very uniform (Figure 6 middle panel). The RMS thickness variation is about $5 \mathrm{~nm}$.

Transmission and reflection tests were carried out in three parts for three wavelength ranges: NIR (0.9-3 $\mu \mathrm{m})$, MIR $(2-25 \mu \mathrm{m})$ and FIR $(20-100 \mu \mathrm{m})$. For the FIR tests, the detector required cooling to $4 \mathrm{~K}$. The results are shown in Figure 8. There is good reflectance of the FIR wavelengths (ranging from $92 \%$ to $95 \%$ over the entire band). The average transmission over the NIR band is $65 \%$ which meets our requirements. A maximum transmission of $76 \%$ was obtained at $\lambda=1.83 \mu \mathrm{m}$. The theoretical maximum from the equivalent circuit model is expected at a lower wavelength $(\sim 1.5 \mu \mathrm{m})$, but this peak is reduced to $62 \%$ because it coincides with the 

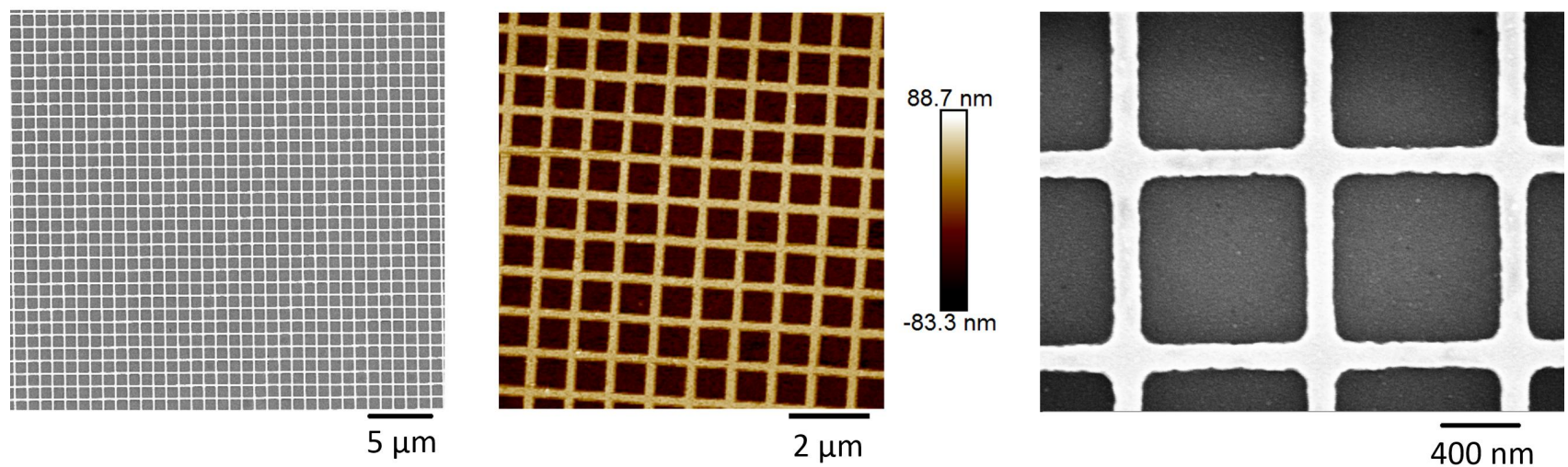

Figure 6. Left: SEM micrograph of the grid pattern. Middle: AFM scan of the metal grid lines giving the thickness profile of the pattern. Right: High zoom SEM image of the inductive grid.

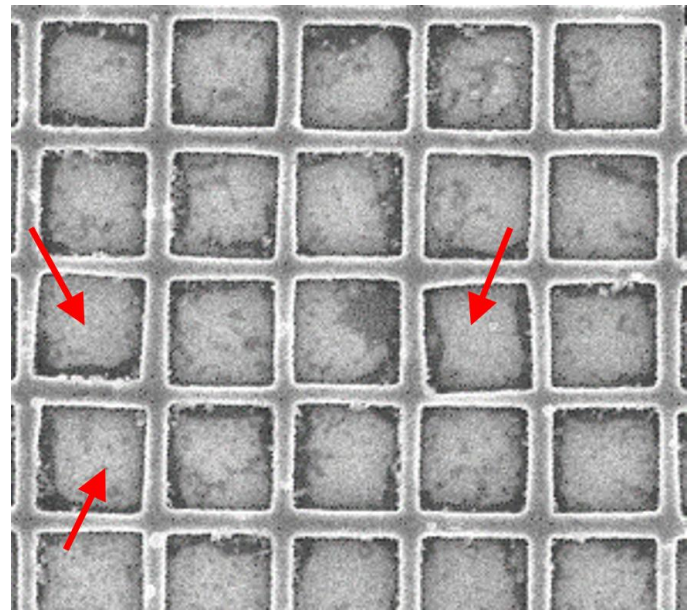

Figure 7. SEM image of a selective region showing pattern defects. The marked tiles are rotated with respect to the grid structure, thereby introducing some local linewidth variations. 

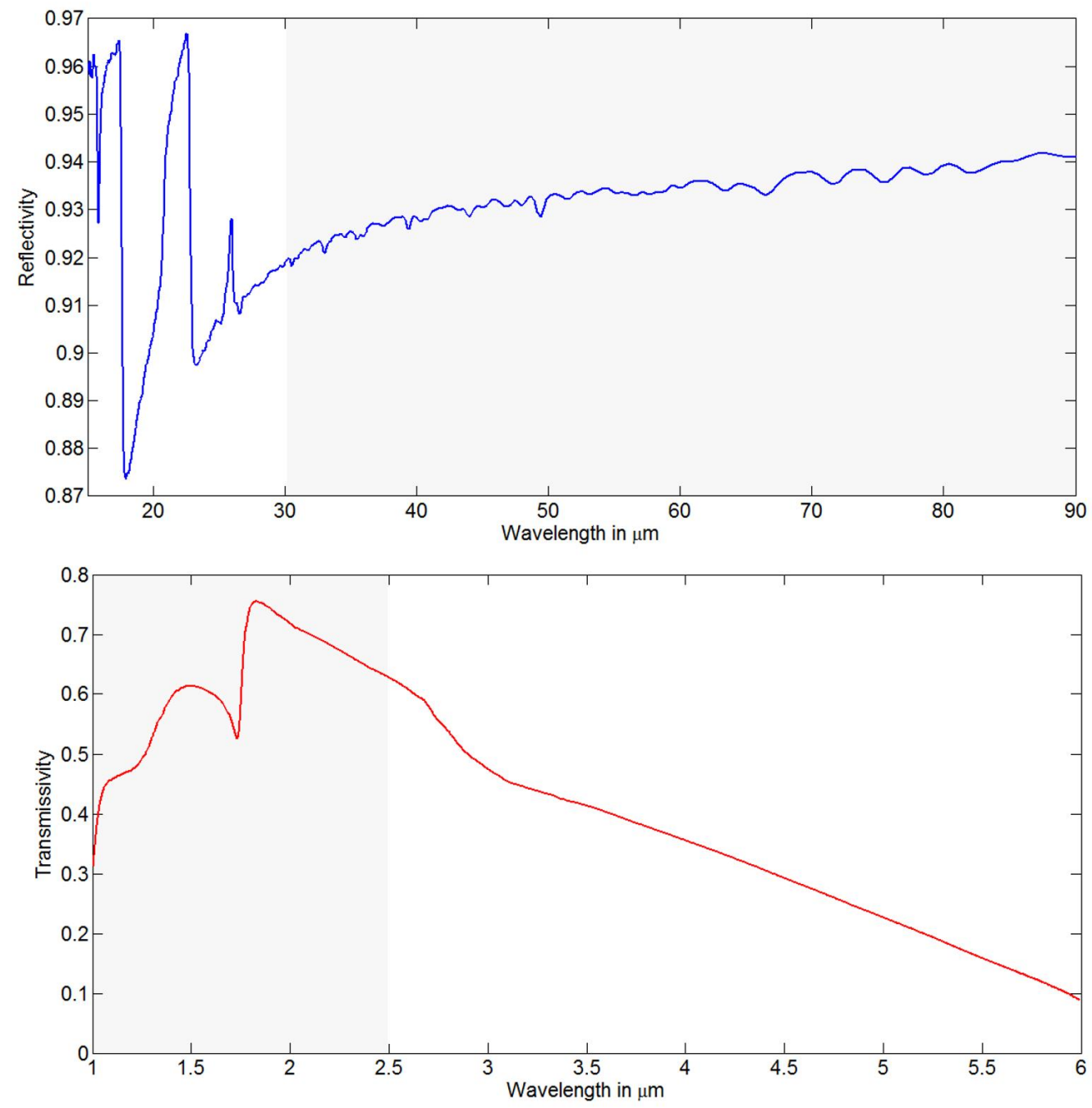

Figure 8. Reflection and Transmission profiles of the NIR/FIR dichroic. The shaded regions indicate BETTII's FIR and NIR bands respectively.

anomalous wavelength regime for this grid pattern. ${ }^{21} \mathrm{~A}$ 'thickness dip' feature is identified at $1.7 \mu \mathrm{m}$ close to the expected wavelength $(1.6 \mu \mathrm{m})$ based on the a/g value of $0.05{ }^{19}$ The tests were carried out at near normal incidence. It is important to operate the dichroic at low incident angles. On increasing the angle of incidence, the 'thickness dip' is more prominent. The non-transmitted light in the NIR wavelengths is reflected by the dichroic. It is subsequently absorbed by the thermal blockers in the FIR optics train immediately after the dichroic.

\section{SUMMARY}

Inductive grids have been used as FIR reflectors for Fabry-Perot Interferometers, ${ }^{26}$ for plasma diagnostics ${ }^{27}$ as output couplers of optically pumped lasers ${ }^{28}$ and in astronomy for blocking excess thermal radiation and defining the observation bands. ${ }^{24,29}$ However, until recently, it was not possible to extend this technique to NIR wavelengths because of fabrication challenges related to the sub-micron length scales of the grid structure.

In this paper, we have described the BETTII requirements for a dichroic NIR/FIR filter. This filter is used to separate NIR radiation used for pointing control from the FIR radiation to be studied by the BETTII doubleFourier interferometer. After considering many options, we designed, fabricated, and tested large area (30 cm 
diameter) dichroic infrared filters suitable for cryogenic applications. These filters efficiently reflect far IR beyond $25 \mu \mathrm{m}$ radiation while transmitting near IR radiation around $2 \mu \mathrm{m}$.

The fabrication of the high density, high resolution metal mesh necessary to attain the desired band separation and performance was achieved using e-beam lithographic techniques. Due to the large areas required for the BETTII filters, the e-beam writing time was significant. Since only two filters were required for BETTII, both filters were made using this technique. For applications requiring larger numbers of filters, other techniques such as imprint lithography would likely be more efficient.

\section{REFERENCES}

[1] Holmes, W. A., Chui, T., Johnson, D., Larson, M., Lysek, M., Paine, C., and Rodriguez, J., "Cooling Systems for Far-Infrared Telescopes and Instruments," in [Astro2010: The Astronomy and Astrophysics Decadal Survey, Technology Development Papers, no. 13], 2010, 13 (Mar. 2009).

[2] Piotrowski, J. and Rogalski, A., "Uncooled long-wavelength infrared photon detectors," in [Proceedings of the SPIE, Volume 5359, p. 10-22 (2004).], 5359, 10-22 (July 2004).

[3] Bernard, J. P., Ade, P., André, Y., Aumont, J., Bautista, L., Bray, N., Bernardis, P. d., Boulade, O., Bousquet, F., Bouzit, M., Buttice, V., Caillat, A., Charra, M., Chaigneau, M., Crane, B., Crussaire, J. P., Douchin, F., Doumayrou, E., Dubois, J. P., Engel, C., Etcheto, P., Gélot, P., Griffin, M., Foenard, G., Grabarnik, S., Hargrave, P., Hughes, A., Laureijs, R., Lepennec, Y., Leriche, B., Longval, Y., Maestre, S., Maffei, B., Martignac, J., Marty, C., Marty, W., Masi, S., Mirc, F., Misawa, R., Montel, J., Montier, L., Mot, B., Narbonne, J., Nicot, J. M., Pajot, F., Parot, G., Pérot, E., Pimentao, J., Pisano, G., Ponthieu, N., Ristorcelli, I., Rodriguez, L., Roudil, G., Salatino, M., Savini, G., Simonella, O., Saccoccio, M., Tapie, P., Tauber, J., Torre, J. P., and Tucker, C., "PILOT: a balloon-borne experiment to measure the polarized FIR emission of dust grains in the interstellar medium," Experimental Astronomy 42, 199-227 (Oct. 2016).

[4] Bintley, D., Holland, W. S., MacIntosh, M. J., Friberg, P., Bell, G. S., Berke, D. A., Berry, D. S., Berthold, R. M., Cookson, J. L., Coulson, I. M., Currie, M. J., Dempsey, J. T., Gibb, A. G., Gorges, B. H., Graves, S. F., Jenness, T., Johnstone, D. I., Parsons, H. A. L., Thomas, H. S., Walther, C., and Wouterloot, J. G. A., "SCUBA-2: an update on the performance of the 10,000 pixel bolometer camera after two years of science operation at the JCMT," in [Proceedings of the SPIE, Volume 9153, id. 91530315 pp. (2014).], 9153 (Aug. 2014).

[5] Naylor, D. A., Baluteau, J.-P., Bendo, G. J., Benielli, D., Fulton, T. R., Gom, B. G., Griffin, M. J., Hopwood, R., Imhof, P., Lim, T. L., Lu, N., Makiwa, G., Marchili, N., Orton, G. S., Papageorgiou, A., Pearson, C., Polehampton, E. T., Schulz, B., Spencer, L. D., Swinyard, B. M., Valtchanov, I., van der Wiel, M. H. D., Veenendaal, I. T., and Wu, R., "In-orbit performance of the Herschel/SPIRE imaging Fourier transform spectrometer: lessons learned," in [Proceedings of the SPIE, Volume 9143, id. $91432 D 15$ pp. (2014).], 9143 (Aug. 2014).

[6] Merrell, W. C., Aslam, S., Brown, A. D., Chervenak, J. A., Huang, W.-C., Quijada, M., and Wollack, E. J., "Compact micromachined infrared bandpass filters for planetary spectroscopy," Applied Optics 51, 3046 (May 2012).

[7] de Graauw, T., Haser, L. N., Beintema, D. A., Roelfsema, P. R., van Agthoven, H., Barl, L., Bauer, O. H., Bekenkamp, H. E. G., Boonstra, A. J., Boxhoorn, D. R., Cote, J., de Groene, P., van Dijkhuizen, C., Drapatz, S., Evers, J., Feuchtgruber, H., Frericks, M., Genzel, R., Haerendel, G., Heras, A. M., van der Hucht, K. A., van der Hulst, T., Huygen, R., Jacobs, H., Jakob, G., Kamperman, T., Katterloher, R. O., Kester, D. J. M., Kunze, D., Kussendrager, D., Lahuis, F., Lamers, H. J. G. L. M., Leech, K., van der Lei, S., van der Linden, R., Luinge, W., Lutz, D., Melzner, F., Morris, P. W., van Nguyen, D., Ploeger, G., Price, S., Salama, A., Schaeidt, S. G., Sijm, N., Smoorenburg, C., Spakman, J., Spoon, H., Steinmayer, M., Stoecker, J., Valentijn, E. A., Vandenbussche, B., Visser, H., Waelkens, C., Waters, L. B. F. M., Wensink, J., Wesselius, P. R., Wiezorrek, E., Wieprecht, E., Wijnbergen, J. J., Wildeman, K. J., and Young, E., "Observing with the ISO Short-Wavelength Spectrometer.," Astronomy and Astrophysics 315, L49-L54 (Nov. 1996). 
[8] Hawkins, G. J., Sherwood, R. E., Djotni, K., and Threadgold, T. M., "Cooled optical filters for Q-band infrared astronomy $(15-40 \mu \mathrm{m}), "$ in [Proceedings of the SPIE, Volume 9912, id. 99123511 pp. (2016).], 9912 (July 2016).

[9] Ade, P. A. R., Pisano, G., Tucker, C., and Weaver, S., "A review of metal mesh filters," in [Society of PhotoOptical Instrumentation Engineers (SPIE) Conference Series], Proceedings of the SPIE 6275, 62750U (June 2006).

[10] Dhabal, A., Rinehart, S. A., Rizzo, M. J., and Mundy, L., "Optics of Balloon Experimental Twin Telescope for Infrared Interferometry (BETTII): delay lines and alignment," in [Proceedings of the SPIE, Volume 9907, id. $99070 T$ (2016)], 9907 (July 2016).

[11] Veach, T. J., Meier, L., Maher, S. F., Wright, R. H., , Keith, C., Rinehart, S. A., Fixsen, D. J., and Dhabal, A., "On-flight performance of an H4RG for the BETTII mission," in [High Energy, Optical, and Infrared Detectors for Astronomy VIII], Proceedings of the SPIE 10709 (June 2018).

[12] Rizzo, M. J., Rinehart, S. A., Alcorn, J. B., Barclay, R. B., Barry, R. K., Benford, D. J., Dhabal, A., Fixsen, D. J., Gore, A. S., Johnson- Shapoval, S., Leisawitz, D. T., Maher, S. F., Mundy, L. G., Papageorgiou, A., Pascale, E., Rau, A., Silverberg, R. F., Taraschi, P., Veach, T. J., and Weinreich, S., "Building an interferometer at the edge of space: pointing and phase control system for BETTII," in [Space Telescopes and Instrumentation 2014: Optical, Infrared, and Millimeter Wave], 9143, 91433H (Aug. 2014).

[13] Vila Hernandez de Lorenzo, J., Rinehart, S. A., Nehmetallah, G., Maher, S. F., Rizzo, M. J., Dhabal, A., Fixsen, D. J., Torres, M. C., Veach, T. J., and Esteves, F. M., "Flight performance of the attitude control system of the balloon experimental twin telescope for infrared interferometry (BETTII)," in [Ground-based and Airborne Telescopes VII], Proceedings of the SPIE 10700 (June 2018).

[14] Bell, E. E., "Measurement of the far infrared optical properties of solids with a michelson interferometer used in the asymmetric mode: Part I, mathematical formulation*," Infrared Physics 6, 57-74 (June 1966).

[15] Ulrich, R., "Far-infrared properties of metallic mesh and its complementary structure," Infrared Physics 7, 37-55 (Mar. 1967).

[16] Saksena, B. D., Pahwa, D. R., Pradhan, M. M., and Lal, K., "Reflection and transmission characteristics of wire gratings in the far infrared," Infrared Physics 9, 43-52 (July 1969).

[17] Sakai, K. and Genzel, L., [Far Infrared Metal Mesh Filters and Fabry-Perot Interferometry], 155-247, Springer US, Boston, MA (1983).

[18] Mitsuishi, A., Otsuka, Y., Fujita, S., and Yoshinaga, H., "Metal Mesh Filters in the Far Infrared Region," Japanese Journal of Applied Physics 2, 574 (Sept. 1963).

[19] Lamarre, J. M., Coron, N., Courtin, R., Dambier, G., and Charra, M., "Metallic mesh properties and design of submillimeter filters," International Journal of Infrared and Millimeter Waves 2, 273-292 (Mar. 1981).

[20] Genzel, L. and Sakai, K., "Interferometry from 1950 to the present.," Journal of the Optical Society of America (1917-1983) 67, 871-879 (Jan. 1977).

[21] Botten, L. C., McPhedran, R. C., and Lamarre, J. M., "Inductive grids in the resonant region: Theory and experiment," International Journal of Infrared and Millimeter Waves 6, 511-575 (July 1985).

[22] Puscasu, I., Boreman, G., Tiberio, R. C., Spencer, D., and Krchnavek, R. R., "Comparison of infrared frequency selective surfaces fabricated by direct-write electron-beam and bilayer nanoimprint lithographies," Journal of Vacuum Science Technology B: Microelectronics and Nanometer Structures 18, 3578-3581 (Nov. 2000).

[23] Walsh, G. F., Osgood, Richard M., I., Ziegler, D., Roy, M., Carlson, J., Belton, L., and Kimball, B. R., "Frequency selective surfaces offer new possibilities as reflectance filters in the NIR/visible spectrum," in [Nanophotonic Materials V. Edited by Gaburro, Zeno; Cabrini, Stefano; Talapin, Dmitri. Proceedings of the SPIE, Volume 7030, article id. 70300E, 10 pp. (2008).], 7030 (Aug. 2008).

[24] Sako, S., Miyata, T., Nakamura, T., Onaka, T., Ikeda, Y., and Kataza, H., "Developing metal mesh filters for mid-infrared astronomy of 25 to 40 micron," in [Advanced Optical and Mechanical Technologies in Telescopes and Instrumentation. Edited by Atad-Ettedgui, Eli; Lemke, Dietrich. Proceedings of the SPIE, Volume 7018, article id. 701853, 10 pp. (2008).], 7018 (July 2008).

[25] Ahmed, Z., Grayson, J. A., Thompson, K. L., Kuo, C. L., Brooks, G., and Pothoven, T., "Large-Area Reflective Infrared Filters for Millimeter/Sub-mm Telescopes," Journal of Low Temperature Physics 176, 835-840 (Sept. 2014). 
[26] Sakai, K., Fukui, T., Tsunawaki, Y., and Yoshinaga, H., "Metallic Mesh Bandpass Filters and Fabry-Perot Interferometer for the Far Infrared," Japanese Journal of Applied Physics 8, 1046 (Aug. 1969).

[27] Walker, B., Baker, E. A. M., and Costley, A. E., "A Fabry-Perot interferometer for plasma diagnostics," Journal of Physics E Scientific Instruments 14, 832-837 (July 1981).

[28] Bruneau, J. L., Belland, P., and Véron, D., "A CW DCN waveguide laser of high volumetric efficiency," Optics Communications 24, 259-264 (Mar. 1978).

[29] Padin, S., Staniszewski, Z., Keisler, R., Joy, M., Stark, A. A., Ade, P. A. R., Aird, K. A., Benson, B. A., Bleem, L. E., Carlstrom, J. E., Chang, C. L., Crawford, T. M., Crites, A. T., Dobbs, M. A., Halverson, N. W., Heimsath, S., Hills, R. E., Holzapfel, W. L., Lawrie, C., Lee, A. T., Leitch, E. M., Leong, J., Lu, W., Lueker, M., McMahon, J. J., Meyer, S. S., Mohr, J. J., Montroy, T. E., Plagge, T., Pryke, C., Ruhl, J. E., Schaffer, K. K., Shirokoff, E., Spieler, H. G., and Vieira, J. D., "South Pole Telescope optics," Applied Optics 47, 4418-4428 (Aug. 2008). 